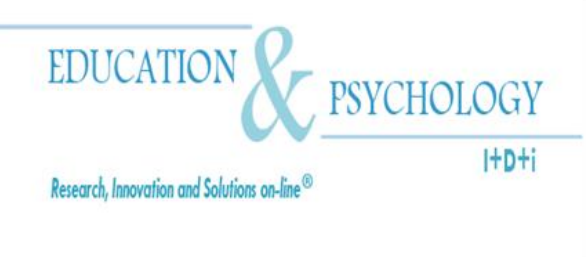

\title{
Autoevaluación: Connotaciones Teóricas y Prácticas. Cuándo Ocurre, Cómo se Adquiere y qué Hacer para Potenciarla en nuestro Alumnado
}

\author{
Ernesto Panadero ${ }^{1}$ y Jesús Alonso-Tapia ${ }^{2}$ \\ ${ }^{1}$ Learning and Educational Technology Research Unit, Faculty of Education, \\ University of Oulu, Finland. \\ ${ }^{2}$ Departamento de Psicología Biológica y de la Salud, Universidad Autónoma \\ de Madrid, España.
}

Finlandia y España

Correspondencia: Ernesto Panadero. Ernesto Panadero Department of Educational Sciences and Teacher Education, Learning and Educational Technology Research Unit (LET). PO BOX 2000, Fin-90014 University of Oulu (Finland). E-mail: ernesto.panadero@gmail.com

C Education \& Psychology I+D+i and Editorial EOS (Spain) 


\section{Resumen}

La autoevaluación es una práctica habitual en las clases en los diferentes niveles educativos. Desafortunadamente un porcentaje elevado de profesores desconoce de qué manera específica afecta al aprendizaje de nuestros alumnos y cómo implementarlo con éxito en las aulas. En este trabajo realizamos un recorrido por las dos corrientes que enfatizan el uso de la autoevaluación (teorías autorregulatorias y evaluación formativa) presentando re-conceptualización de lo que significa autoevaluación. Posteriormente se analiza cuáles son las relaciones entre la autoevaluación y el uso de estrategias de aprendizaje de los alumnos y cómo se adquiere esta capacidad. A continuación se presentarán estrategias pedagógicas que fomentan una buena implementación en el aula para que los profesores podamos ayudar a nuestros alumnos a adquirir la capacidad de autoevaluarse.

Palabras Clave: autoevaluación, autorregulación, evaluación formativa, rúbricas, guiones de evaluación, criterios de evaluación.

Recepción: 20/04/13 Aceptación inicial: 15/05/2013 Aceptación final: 15/07/13 


\title{
Self-assessment: Theoretical and Practical Connotations. When it Happens, How is it Acquired and what to do to Develop it in our Students
}

\begin{abstract}
Self-assessment is one of the most usual activities in classroom from all different educational levels. However a high percentage of teachers report not knowing how self-assessment influence students' learning or how to implement it successfully in their classrooms. In this article two different lines of research concerning self-assessment will be analyzed (self-regulation and formative assessment) along with a new conceptualization of what self-assessment is. Later the relationship between self-assessment and the use of learning strategies by the students will be examined explaining how this skill can be acquired. In the last sections it will be analyzed how the use of different pedagogic strategies can enhance a successful implementation of self-assessment in classrooms.
\end{abstract}

Keywords: self-assessment, self-regulation, formative assessment, rubrics, scripts, assessment criteria.

Received: 04/20/13 Initial acceptance: 05/15/13 Final acceptance: 07/15/13 


\section{Introducción}

En el presente trabajo se analizan diferentes perspectivas sobre la autoevaluación con la intención de visualizar la complejidad teórica de esta estrategia, y de simplificar su uso a través de recomendaciones pedagógicas pertinentes. Para conseguir este objetivo se empieza distinguiendo entre autoevaluación como estrategia de aprendizaje que el alumno puede activar, y autoevaluación como estrategia pedagógica a través de la cual el profesor pide a sus alumnos que reflexionen sobre su trabajo. Esta distinción, proveniente de diferentes tradiciones investigadoras, nos servirá para reflexionar sobre los objetivos de cada aproximación teórica para llegar a una visión compartida. En segundo lugar, se presenta la definición de autoevaluación, explicando cada uno de los elementos que la conforman de forma detallada para visualizar la importancia de que sea esa y no otra la definición. En tercer lugar, se pasa a exponer la relación que existe entre autoevaluación y aprendizaje autorregulado -el uso de estrategias de aprendizaje por parte del alumno-. Para ello, se hace un recorrido por lo que dicen las diferentes teorías sobre autorregulación, se recoge la evidencia empírica que existe sobre esta relación, se expone en qué fases de la autorregulación influye la autoevaluación, y se pone un ejemplo argumentado para mayor concreción. Posteriormente, se analiza cómo se adquiere esta capacidad para acto seguido presentar las condiciones pedagógicas que, si el docente procura que estén presentes, fomentan la autoevaluación en los alumnos. Finalmente se cierra el artículo con una breve introducción a las diferentes intervenciones que se utilizan en el aula para fomentar la autoevaluación.

\section{Algunas consideraciones previas}

Un aspecto clave antes de definir la autoevaluación es diferenciar dos vertientes teóricas que la incluyen. Dentro de la primera vertiente se entiende la autoevaluación como un proceso instruccional que el profesor utiliza como recurso pedagógico. "Vamos a evaluar nuestro trabajo" es una frase que se puede oír en el aula. Y es que un alto porcentaje de profesores de primaria, secundaria y universidad reconocen utilizar la autoevaluación como proceso instruccional (Noonan y Duncan, 2005; Panadero, en preparación). Entendida como proceso instruccional, la autoevaluación forma parte de la evaluación formativa ('formative assessment' en inglés) junto a otros tipos de evaluación (por ejemplo la evaluación entre pares) (Black y Wiliam, 1998).

La segunda vertiente que incluye la autoevaluación arranca de las teorías sobre autorregulación: Es el control que el sujeto realiza sobre sus pensamientos, acciones, emociones y 
motivación a través de estrategias personales para alcanzar los objetivos que ha establecido (Zimmerman, 2000, pág. 14). En esta vertiente la autoevaluación es entendida como un proceso que el alumno realiza para autorregular su aprendizaje. Como ilustración, en el modelo de autorregulación de Zimmerman que se presenta en la Figura 1 puede verse que la la autoevaluación constituye uno de los procesos autorregulatorios.

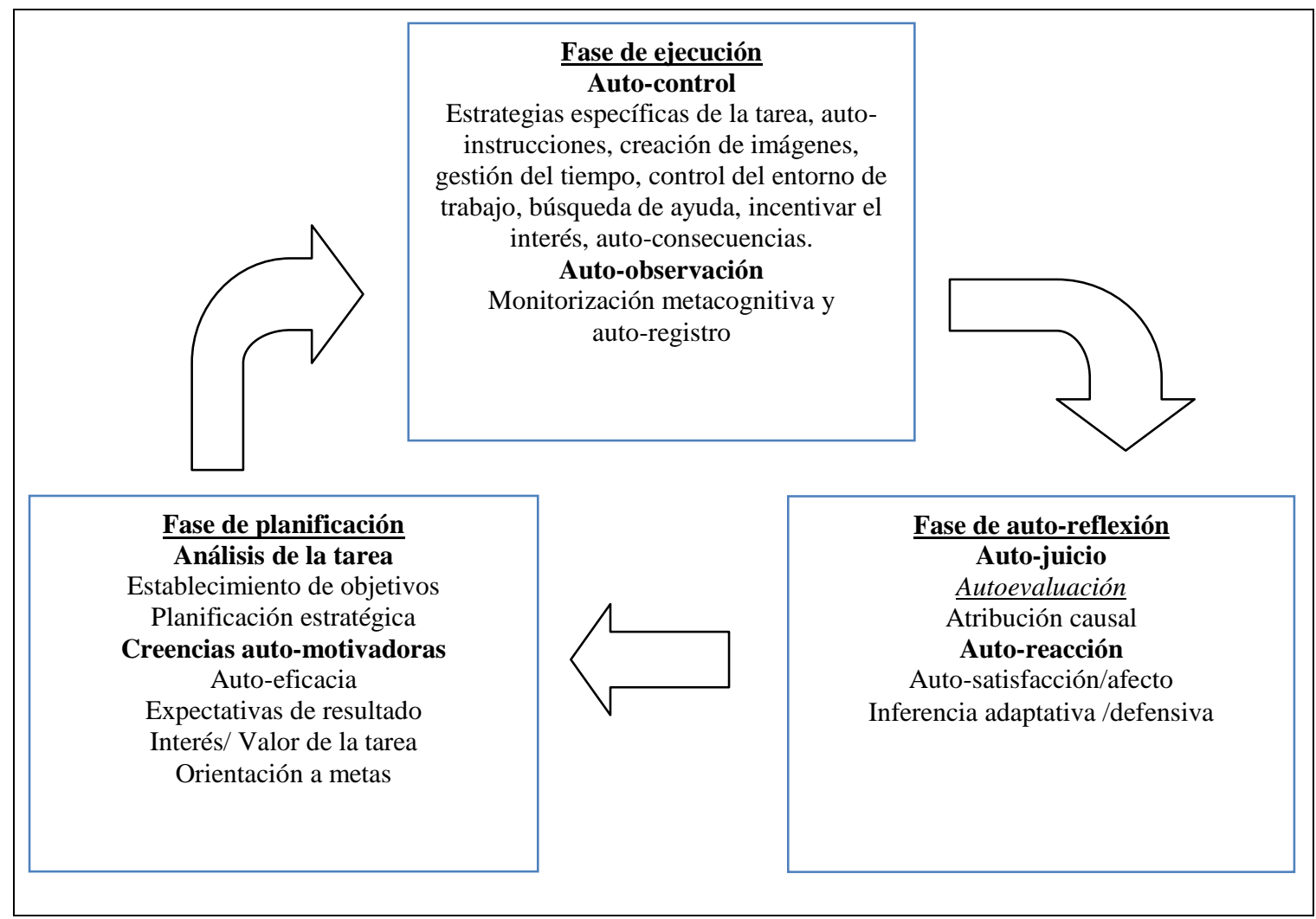

Figura 1. Fases y procesos de la autorregulación (Zimmerman y Moylan, 2009).

Aunque la diferencia entre estas dos vertientes parece obvia, no resulta patente en muchos trabajos sobre autoevaluación, siendo habitual que se utilice una vertiente u otra pero rara vez se presentan de forma conjunta. Los profesores a veces tienen una visión superficial del valor pedagógico de la autoevaluación, considerándola un mero apoyo instruccional sin entender la importancia real que conlleva para el aprendizaje de los alumnos (Boud y Falchikov, 1989; Tan, 2012). Sin embargo, desde nuestro punto de vista el profesor al fomentar la autoevaluación debería emplearla como estrategia instruccional cuyo objetivo final debería ser guiar a los alumnos para que aprendiesen a autoevaluarse, de modo que este aprendizaje repercutise en su autorregulación. En otras palabras, utilizar la autoevaluación no es "solo" una decisión pedagógica sin mayores repercusiones. Los profesores deberían ser cons- 
cientes de que fomentarla implica entrenar tal proceso de forma explícita, intentando garantizar que los alumnos aprendan a autoevaluarse, es decir potenciar toda una serie de procesos metacognitivos -planificación, supervisión y evaluación- (Winne y Hadwin, 1998). Compartir esta conceptualización es importante pues los profesores que utilizan la autoevaluación como apoyo instruccional tienen a menudo dudas sobre su utilidad, y sobre la exactitud de las valoraciones de los alumnos (Noonan y Duncan, 2005; Panadero, en preparación). Cómo se puede conseguir hacer un uso efectivo de la autoevaluación como estrategia instruccional es algo que se verá más adelante. Pero, lo que no hay que olvidar, como explica Boud (1995a), es que "la autoevaluación consiste en que los alumnos desarrollen sus habilidades de aprendizaje. No es sólo una técnica de evaluación entre otras" (pág. 15).

En resumen, cuando se parte de una visión más pedagógica sobre la autoevaluación, la que suele subyacer a los trabajos sobre evaluación formativa (Black yWiliam, 1998, 2009), no se suele hacer explícito el proceso interno que se entrena con ella. Por el contrario, cuando la autoevaluación se conceptualiza como un proceso autorregulatorio, como suele ocurrir en trabajos sobre autorregulación (Puustinen y Pulkkinen, 2001), los autores se centran en el proceso interno, y no se suelen hacer explícto que es un recurso instruccional que el profesor puede/debe utilizar y fomentar, excepto en contadas excepciones (Paris y Paris, 2001).

Aquí se presentarán las implicaciones para el aprendizaje y para la práctica docente desde las dos vertientes, de forma que el uso de la estrategia instruccional tendrá como objetivo entrenar el proceso interno autorregulatorio. Con este fin expondremos primero qué se entiende por autoevaluación y su relación con la autorregulación para, posteriormente, exponer las condiciones pedagógicas que fomentan en el aula la adquisición de esta habilidad.

\section{Naturaleza de la autoevaluación}

Una de las definiciones clásicas de autoevaluación la describe como "la participación del alumno juzgando su propio aprendizaje, específicamente sus logros y los resultados del mismo" (Boud y Falchikov, 1989, pág. 529). Aunque es una definición muy extendida, en este trabajo utilizamos una definición con algunas características innovadoras que aquella no contiene y que pasamos a exponer.

Desde nuestro punto de vista, la autoevaluación consiste en: "la valoración cualitativa de la ejecución, esto es, proceso de aprendizaje y del producto final del mismo, valoración realzada a partir de unos criterios preestablecidos y modulada por los niveles de perfección 
que el alumno desee alcanzar" (Panadero, 2011, pág. 78). A continuación, analizamos la definición con detenimiento, reflexionando sobre lo que significa cada elemento de la misma.

La primera parte de la definición establece que la autoevaluación es un "valoración cualitativa". Esto implica que autoevaluarse no es una mera valoración cuantitativa o "ponerse nota”. Esta última práctica, que se denominó autoevaluación durante mucho tiempo, cuando su nombre más adecuado dbería haber sido auto-calificación (self-grading, self-evaluation o self-marking en inglés) (Andrade y Valtcheva, 2009), no es una buena estratégica pedagógica pues no garantiza la reflexión sobre el trabajo realizado (Alonso-Tapia y Panadero, 2010; Panadero, Alonso-Tapia y Huertas, 2012). Por lo tanto, el hecho de que la definición haga referencia a la "valoración cualitativa" implica que lo importante en la autoevaluación no es la autocalificación, sino la comprensión por parte del alumno del proceso seguido, comprensión que posibilita aprender de los errores y aciertos. La autoevaluación es, pues, un proceso reflexivo que se apoya en criterios preestablecidos.

Otro de los aspectos relevantes de la definición propuesta en este trabajo es que la autoevaluación es la valoración “de la ejecución”, esto es, del proceso de aprendizaje y del producto final del mismo. ¿Por qué es relevante este aspecto? Muchas definiciones consideran que la autoevaluación sólo ocurre al finalizar la actividad, cuando el alumno acaba el trabajo y evalúa el producto final. Sin embargo, la autoevaluación también ocurre durante el proceso de aprendizaje, en la medida en que el alumno supervisa lo que va haciendo y lo compara con sus criterios de procedimiento (Greene y Azevedo, 2007; Winne y Hadwin, 1998). Además, una correcta autoevaluación influye también en la fase de planificación (Boud, 1995b), hecho por el que se recomienda explicitar a los alumnos los criterios de evaluación desde el principio de la actividad (Andrade, 2010; Andrade y Valtcheva, 2009; Boekaerts y Corno, 2005). La razón de esta recomendación es que, al tener más claros los objetivos, los alumnos autorregulan mejor su trabajo (Boekaerts y Cascallar, 2006). En resumen, la autoevaluación influye en todas las etapas del proceso autorregulatorio, actuando sobre el aprendizaje de forma recursiva durante la planificación, la realización y la evaluación final del mismo (Zimmerman y Moylan, 2009).

Otro elemento novedoso de la definición es la inclusión de los "criterios de evaluación". Estos criterios son las directrices específicas bajo las que se evaluará el trabajo tanto por parte del profesor como del alumno (Goodrich, 1996). Los criterios pueden ser establecidos de tres formas. Primero, de forma externa, si los establece el profesor; segundo, pueden tener origen 
externo pero estar aceptados internamente, por ejemplo si el profesor los discute y negocia con los alumnos; y, tercero, pueden ser de origen interno, cuando el sujeto reflexiona sobre las características que debe tener su trabajo y establece sus propios criterios. Cuanta mayor sensación de autonomía en la elección de los criterios, mayor motivación habrá por alcanzarlos, tal y como ocurre con otras metas (Deci y Ryan, 1985). Finalmente, estos criterios, al dejar claros los objetivos a alcanzar, son fundamentales para la autorregulación guiando al alumno que activaría las estrategias de aprendizaje de una forma más selectiva (Andrade y Valtcheva, 2009; Boekaerts y Corno, 2005).

No obstante, hay mucha polémica en torno al hecho de hacer explícitos estos criterios. La investigación indica que cuando el profesor los hace explícitos mejora el aprendizaje (Andrade, 2010; Andrade y Valtcheva, 2009; Boekaerts y Corno, 2005). Sin embargo, muchos profesores no quieren explicitarlos pues consideran que es "contar el secreto" de qué van a preguntar. Para evitar esta oposición, es importante enfatizar que lo que se recomienda hacer explícito no es el contenido por el que se va a preguntar, sino bajo qué criterios se va a evaluar lo que se va a preguntar -cómo se corregirá-. Esto es, es explicar a los alumnos las "reglas del juego" para que sepan cómo se va evaluar su trabajo de modo que tengan una mayor sensación de control, lo que permitiría que aumentase su interés en la tarea y que pudiesen autorregular su realización adecuadamente. De hecho los alumnos que tienen claras y bien estructuradas sus metas tienen más posibilidades de regular su motivación y su esfuerzo (Boekaerts y Cascallar, 2006; Boekaerts y Corno, 2005).

La última parte de la definición hace referencia a los niveles de perfección que el alumno quiere alcanzar en su trabajo. El alumno fija unos objetivos atendiendo al nivel de perfección que quiere alcanzar en una actividad concreta, nivel que puede depender del tipo de tarea -su naturaleza, su importancia, su nivel de dificultad o su ambigüedad. Por ejemplo: un alumno al que le resulten difíciles las matemáticas intentará alcanzar un nivel de realización que le permita aprobar la asignatura. Sin embargo, un alumno que desee obtener un sobresaliente intentará alcanzar un nivel de realización excelente. Estos niveles también influyen en la autoevaluación pues un alumno, aun siendo consciente de que su trabajo es mejorable, puede darse por satisfecho al llegar al nivel que fijó al planificar la tarea. 


\section{Papel de la autoevaluación en el proceso de autorregulación}

Como se está argumentado a lo largo del manuscrito, la autoevaluación es un proceso clave para la autorregulación, porque supone reflexionar y tomar conciencia del proceso de aprendizajre y del resultado del mismo (Paris y Paris, 2001; Puustinen y Pulkkinen, 2001; Winne y Hadwin, 1998; Zimmerman y Moylan, 2009). Esta reflexión sobre lo que se ha hecho permite repetirlo si ha sido realizado de forma correcta, hasta llegar a alcanzar un modelo de ejecución experto. Si, por el contrario, la ejecución ha sido incorrecta, la autoevaluación permite tomar conciencia de los errores y subsanarlos. Por este motivo, la autoevaluación permite mejorar las ejecuciones futuras de la actividad y, además, favorece la transferencia de los modos de actuación a otros aprendizajes (Boud, 1995a). Las diferentes teorías sobre la autorregulación han puesto de manifiesto la relación entre autorregulación y autoevaluación y la evidencia empírica en que se apoya, relación y apoyo que pasamos a exponer.

A nivel teórico, Puustinen y Pulkkinen (2001) en su revisión concluyeron que las cinco principales teorías de autorregulación consideran la autoevaluación un proceso clave. En otra revisión teórica, Zimmerman (2001) señalaba el mismo hecho analizando siete teorías, sólo dos de las cuales estaban incluidas en la revisión de Puustinen y Pulkkinen. Así mismo, si se analizan las diferentes teorías de forma independiente, incluso aquellas que no estaban incluidas en las revisiones, se puede encontrar en todas ellas la importancia de la relación entre autoevaluación y autorregulación (e.g. Belfiore y Hornyak, 1998; McCaslin y Hickey, 2001; McCombs, 2001; Paris y Paris, 2001). Estos últimos llegan a afirmar: "How can academic assessments of learning be infused with the same passion and autonomy? Self-assessment may be the key. Self-assessment includes all three domains of SRL: cognitive, motivational, and affective." (Paris y Paris, 2001; pág. 95). Así pues, desde las teorías de la autorregulación la autoevaluación se conceptualiza como un proceso fundamental. Pero además, es preciso señalar que también los teóricos de la autoevaluación enfatizan el papel que ésta tiene en la autorregulación (Andrade, 2010; Bannert, 2009; Boud, 1995c).

A nivel empírico, hay evidencia que pone de manifiesto la relación entre autoevaluación y autorregulación. Los alumnos con alta habilidad para autorregular su aprendizaje autoevalúan su trabajo con más eficiencia y en más ocasiones que los alumnos con baja habilidad para autorregularlo (Lan, 1998). Asimismo, los alumnos con éxito académico supervisan y evalúan su pensamiento más que los que no lo tienen (Biemiller y Meichenbaum, 1992). Además hay evidencia de que las intervenciones basadas en estrategias autoevaluatorias (monitorización y 
evaluación) mejoran la autorregulación (Bannert, 2009; Dignath, Büttner, y Langfeldt, 2008; Kostons, van Gog, y Paas, 2009; Kramarski y Michalsky, 2009, 2010; Kramarski y Zeichner, 2001; Sitzmann y Ely, 2010; van den Boom, Paas, Merriënboer, y van Gog, 2004; Wirth, 2009). Así mismo, el modelo interactivo de autorregulación propuesto por de la Fuente y cols. (de la Fuente y Justicia, 2007, de la Fuente et al., 2012) también fundamenta conceptualmente esta relación. Por lo tanto, dado que aprender a autoevaluarse tiene efectos positivos sobre la autorregulación, su enseñanza debería ser un objetivo instruccional. Sin embargo, para favorecer el éxito de las intervenciones de los profesores orientadas a mejorar la autoevaluación y la autorregulación, conviene conocer en profundidad cómo funciona la autoevaluación en relación con el proceso autorregulatorio, punto que se analiza en la siguiente sección.

\section{¿Cuándo ocurre la autoevaluación?}

Como ya se ha señalado, cuando Zimmerman y Moylan (2009) conceptualizan el proceso de autorregulación, sitúan la autoevaluación en la última fase, la de auto-reflexión. Sin embargo, hay teorías autorregulatorias que consideran que la auto-monitorización -que ocurre en la fase de ejecución- tiene una importancia capital en la autoevaluación, ya que es una parte esencial de la misma (Greene y Azevedo, 2007; Winne y Hadwin, 1998), aspecto que los autores de este trabajo suscriben junto a otros autores cuyos trabajos se centran en la evaluación y la autoevaluación (Kostons et al., 2009; Segers, Dochy, y Cascallar, 2003). Otros autores van más allá, recomendando que cuando se enseñe a los alumnos a autoevaluar su trabajo se intervenga desde la fase de planificación-(Andrade y Valtcheva, 2009; Boekaerts y Corno, 2005; Boud, 1995b). La razón es que, si se enseña a los alumnos a autoevaluarse desde la fase de planificación, pueden seguir haciéndolo supervisando la ejecución de la tarea durante la fase de realización de la misma, autosupervisión que les puede permitir detectar si su actuación es adecuada o no y modificar su actuación si detectan que no es efectiva para su aprendizaje. Al mismo tiempo, este proceso activa la motivación de los alumnos, pues la toma de conciencia de lo que ocurre se experimenta como progreso, experiencia que facilita el interés por la tarea y la sensación de autoeficacia, características que favorecen su aprendizaje. A continuación vamos a argumentar porqué la autoevaluación influye durante las fases de planificación y ejecución de la tarea en la autorregulación y el aprendizaje.

¿Por qué la enseñanza adecuada de la autoevaluación, puede influir en el proceso de aprendizaje desde la fase de planificación de la tarea? Como ya se ha explicado, para fo- 
mentar la autoevaluación se recomienda entregar a los alumnos los criterios de evaluación al principio de la actividad, ya que al disponer de los mismos pueden planificar estratégicamente su actuación (Andrade y Valtcheva, 2009; Boekaerts y Cascallar, 2006).

¿Por qué ocurre la autoevaluación durante la fase de ejecución? Imaginemos a un alumno que está supervisando sus acciones durante la ejecución de una tarea. Este alumno duda si está realizando correctamente la actividad, así que revisa los criterios que le ha dado el profesor y toma conciencia de que lo que está haciendo no es adecuado y decide modificar su actuación (Ver Cuadro 1). Lo que este alumno ha hecho es autoevaluarse durante el proceso de ejecución de la tarea, hecho que ocurre constantemente a lo largo de las mismas y no solo al acabarlas. En suma, los alumnos no sólo evalúan el producto final del proceso de aprendizaje, sino también la forma de alcanzar ese producto.

El hecho de que la autoevaluación pueda ocurrir también durante el proceso de aprendizaje tiene importantes implicaciones pedagógicas. Para mejorar el resultado la autoevaluación, ésta no se tiene que centrar sólo en el producto final del aprendizaje, sino que se debe también centrar en el proceso, pues no hacerlo puede dar lugar a un resultado erróneo, hecho que supone que el esfuerzo realizado no ha sido eficaz, ya que se tiene que rehacer el trabajo, lo cual disminuye la motivación del alumno (Alonso-Tapia, 2005).

Tabla 1. Ejemplo de autoevaluación del proceso y del producto

\begin{tabular}{|c|c|}
\hline 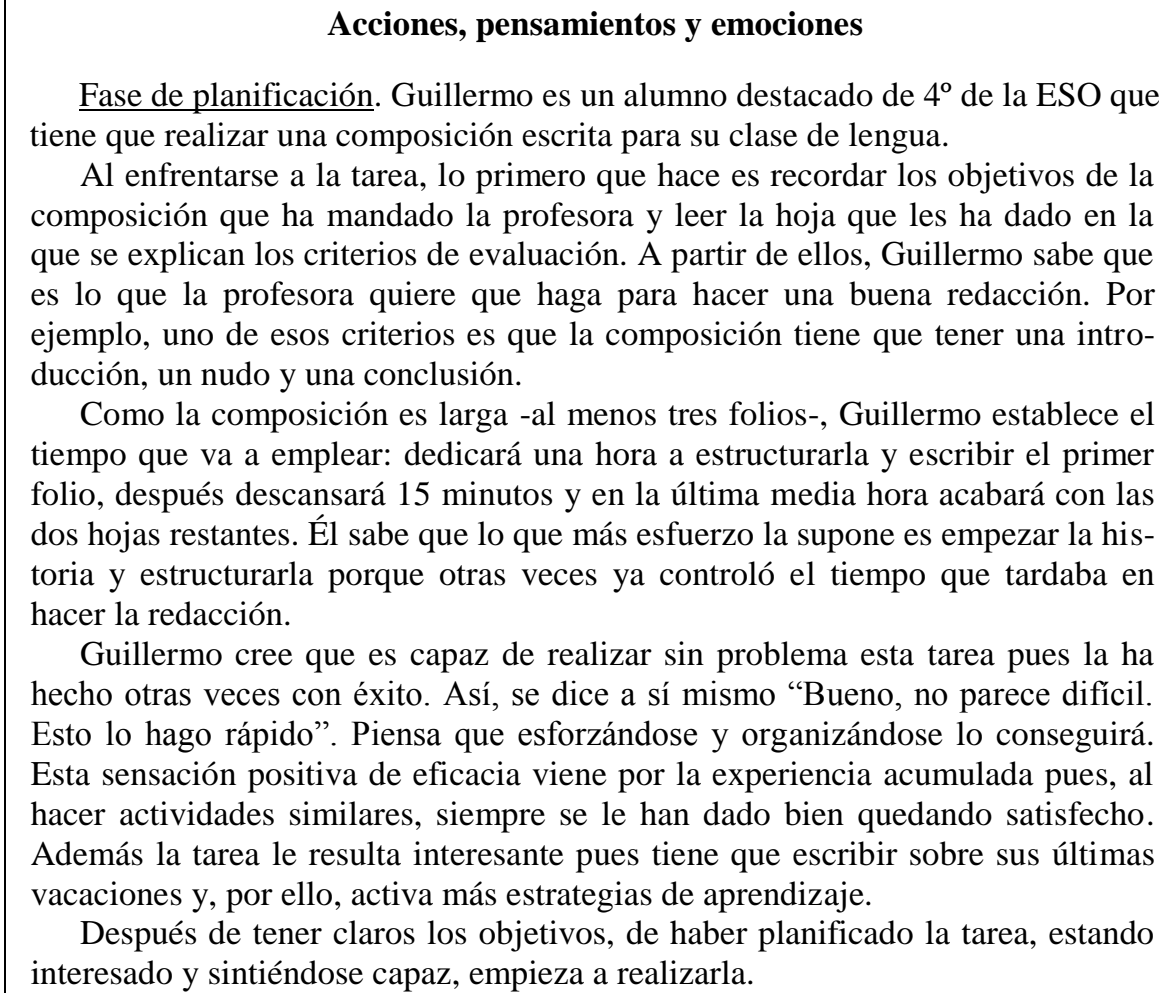 & $\begin{array}{l}\text { Planificación: Planificar } \\
\text { la actividad y el tiempo } \\
\text { que va a dedicar para te- } \\
\text { ner control sobre sus } \\
\text { avances }\end{array}$ \\
\hline
\end{tabular}


Fase de ejecución. Durante la actividad, Guillermo va recordando las vacaciones para elegir qué contar. Presta atención a los acentos pues últimamente le han restado puntos. También va recordando los objetivos para hacer un trabajo que se ajuste a lo pedido.

Cuando lleva media hora trabajando entra su hermano mayor y le dice que si quiere jugar con él al ordenador. Guillermo se siente muy tentado sin embargo, se dice a sí mismo que cuando acabe podrá jugar tranquilamente. Su hermano se va de la habitación pero la idea de irse a jugar le ronda. Para que no le moleste se imagina a sí mismo jugando cuando haya acabado. De esta forma, consigue volver a la tarea y concentrarse de nuevo.

Hay un momento en el que se para a pensar si ha hecho una introducción correcta para la composición. Va a comprobar los criterios de la profesora y lee que se debe presentar el tema y los personajes que aparecerán. En ese momento se da cuenta de que todavía no ha hablado de su familia con quien hizo el viaje, así que re-escribe la introducción para que se ajuste a los objetivos.

Fase de auto-reflexión. Una vez acabada la tarea, Guillermo revisa su trabajo de nuevo, pero ahora con el producto acabado. Para ello, recuerda los criterios que le dio la profesora y comprueba que los ha alcanzado, llegando a la conclusión de que es un buen trabajo. Si de él dependiese se pondría una calificación muy alta. Se siente satisfecho y espera que la profesora coincida con él.

Dos días después recibe la composición calificada con una buena nota. Guillermo lo atribuye a su trabajo y esfuerzo.
Ejecución: Recordar las vacaciones es una estrategia para producir contenidos. Examinar la acentuación implica supervisar la tarea. Ambas permiten regular el trabajo.

Auto-control (creación de imágenes): Decirse a sí mismo que podrá jugar al finalizar e imaginarse jugando son estrategias que permiten regular la concentración.

Autoevaluación del proceso: Comprobar sí actúa según lo previsto implica autoevaluarse y autorregular la actividad. Es un ejemplo de que la autoevaluación ocurre antes de llegar al producto final.

Autoevaluación del producto: Comparar lo que hace con los criterios de evaluación pero, en esta ocasión, tras acabar la composición implica autoevaluación y autorregulación.

En conclusión, la autoevaluación es un proceso fundamental para autorregular el aprendizaje, ya que implica la toma de conciencia de los objetivos de la tarea y la supervisión del progreso que el alumno hace con respecto a los mismos. Ahora bien, si la autoevaluación es realmente importante en el aprendizaje, cabe preguntarse ¿cómo se adquiere esta capacidad?, pues si se comprende cómo se adquiere, se podrá proponer condiciones e intervenciones para favorecerla realmente efectivas.

\section{¿Cómo se adquiere la capacidad de autoevaluación?}

La adquisición de las competencias autorregulatorias requiere de esfuerzo por parte del alumno durante un tiempo tanto más largo cuanto más compleja es la competencia a adquirir, (en el ámbito académico serían competencias: aprender a resumir, a realizar un mapa conceptual, una presentación oral de un trabajo, etc). Y, precisamente, la autoevaluación de diferentes tareas es una competencia compleja, siendo por ello importante que el profesor ayude a 
adquirirla (Boud, 1995c; Brown y Harris, 2013; Dochy, Segers, y Sluijsmans, 1999; Griffee, 1995). Sin embargo, la ayuda no siempre es adecuada como se presenta en el siguiente ejemplo.

Imaginemos un alumno que está realizando por primera vez una división. Realiza la actividad siguiendo los pasos que le ha indicado el profesor. Cuando llega a la solución le entrega el ejercicio al profesor para que lo corrija. Éste lo corrige diciéndole que lo ha hecho mal y le da la solución correcta sin explicarle nada más. De esta forma, el alumno no interioriza el criterio que le permitiría autoevaluarse, hecho que le lleva a depender de la evaluación del profesor para valorar la calidad de su aprendizaje y su actuación en el futuro.

Para ayudar al desarrollo de la autoevaluación es mejor -más efectivo- actuar como en el ejemplo siguiente:

En otra situación, el alumno realiza el ejercicio y, cuando le pide al profesor que lo corrija, éste le entrega una hoja con un modelo del ejercicio realizado por pasos para que compare y decida si tiene que cambiar algo. El alumno compara su ejercicio con el modelo dándose cuenta que se ha equivocado en un paso y modifica su ejercicio hasta alcanzar la respuesta correcta.

En este caso el profesor sí ha entrenado al alumno para que evalúe su propio trabajo, comparando su ejecución con un modelo y corrigiendo los errores cometidos. En consecuencia, si esta habilidad se trabaja en clase, este alumno se acostumbrará a autoevaluarse y de esta forma será más autónomo.

Desafortunadamente, no todos los alumnos son capaces de autoevaluar su trabajo de esta forma. Por ello, hay que enseñar explícitamente a hacerlo para que todos tengan oportunidad de aprender, lo cual hace imprescindible la intervención de un profesor que enseñe cómo se hace. La importancia de enseñar y fomentar la autoevaluación radica en su valor para el desarrollo de la autorregulación en el alumno, ya que necesitará ambas habilidades cuando el nivel de exigencia de la tarea aumente en los cursos posteriores (Dignath et al., 2008). Para que este cambio en la demanda de la tarea no sea tan marcado, es necesario 
que el alumno reciba la ayuda del profesor y vaya formando su habilidad en autoevaluación desde temprana edad (Dochy et al., 1999; Griffee, 1995).

Para entender mejor como fomentar la autoevaluación y la autorregulación es necesario conocer las condidiones que facilitan su adquisición. Aunque no hay estudios sobre la adquisición de la capacidad de autoevaluación, sí los hay sobre la capacidad de autorregulación, de la que aquella es un proceso básico. La idea que subyace a ambas teorías de adquisición es que los alumnos necesitan modelos, experiencia y/o intervención directa para aprender. Así pues, los alumnos pueden aprender a autoevaluar su trabajo con enseñanza directa del profesor, a través de observación, emulación, práctica, en actividades de grupo, etc. El objetivo de las intervenciones es transmitirles un modelo de autoevaluación para que ellos lo apliquen.

Por ello toca preguntarse, ¿qué podemos hacer para ayudar a nuestros alumnos a autoevaluar su trabajo de modo regular? Para ofrecer respuesta, en la siguiente sección, examinamos qué condiciones son necesarias para potenciar el uso de la autoevaluación.

\section{Condiciones que fomentan la autoevaluación}

Aunque incluso alumnos muy jóvenes son capaces de autoevaluar su trabajo, no siempre lo hacen; quizá porque una o más de las condiciones necesarias no se dan en sus entornos de aprendizaje (Andrade y Valtcheva, 2009). En relación con estas condiciones es posible distinguir entre condiciones propiamente dichas -hechos que si no se dan impiden la autoevaluación- y ayudas instruccionales que el profesor puede proporcionar para enseñar a sus alumnos a autoevaluarse y fomentar la autoevaluación (Goodrich, 1996). Las condiciones necesarias para la autoevaluación son (Goodrich, 1996):

a) Conciencia del valor de la autoevaluación: a menos que los alumnos sean conscientes de la utilidad de autoevaluarse no activarán este proceso pues supone un coste. Por ello, es importante que comprendan que la autoevaluación es una habilidad muy importante para su aprendizaje.

b) Tener acceso a los criterios en los que se basa la evaluación: los alumnos han de conocer estos criterios porque son los mismos bajo los que han de autoevaluarse. Es más, se recomienda que tengan acceso a ellos desde el principio de la actividad, como ya se ha explicado reiteradamente a lo largo del artículo (Andrade y Valtcheva, 2009). 
c) Especificidad de la tarea a evaluar: si la tarea resulta muy amplia o no está claramente definida resulta complicado autoevaluar su realización. Por ello, es recomendable que los profesores, teniendo en cuenta este hecho, intenten elegir tareas que bien delimitadas y con pasos definidos, al menos en las primeras ocasiones en que intenten enseñar a sus alumnos a autoevaluar su trabajo.

En cuanto a las ayudas instruccionales mediante las que se ha intentado fomentar la autoevaluación, ayudas que buscan facilitar las condiciones anteriores, son las siguientes:

1) “Modelado" del proceso de autoevaluación. Dado que los alumnos aprenden muchas veces a través de la observación de un experto, es recomendable que vean a un modelo autoevaluarse en voz alta, por ejemplo, a su profesor o un compañero de clase que tenga experiencia en la competencia que se está entrenando. Este tipo de modelado (thinking aloud) resulta especialmente efectivo si primero lo realiza un modelo y después se pide al alumno que lo realize, exprensando en voz alta todos los pasos que va siguiendo así como la lógica tras ellos. De esta forma el profesor puede detectar y corregir errores a lo largo de la ejecución.

2) Instrucción y asistencia directa para la autoevaluación. Es necesario que los alumnos reciban instrucciones hasta que comprendan el procedimiento y sean capaces de ser autónomos. Esto será especialmente así, si la actividad a autoevaluar les resulta desconocida, pues en ese caso desconocerán los criterios mediante los que evaluarla y cómo aplicarlos. Pero además, también han de recibir retroalimentación sobre su propia autoevaluación para que sepan si lo están haciendo adecuadamente y, en caso negativo, qué aspectos deben mejorar (Dochy et al., 1999; Griffee, 1995). Para enseñar de modo adecuado cómo autoevaluar el propio trabajo es, pues, necesario e importante que los profesores sepana cómo hacerlo.

3) Pistas que ayuden a saber cuándo es apropiado autoevaluar. Ofrecer indicaciones del momento en que han de autoevaluar. Aquí de nuevo es útil el modelado del profesor para que los alumnos visualicen cuándo hacerlo.

4) Práctica. Con observar una vez cómo se puede evaluar el propio trabajo no basta para aprender a hacerlo. Es preciso que el profesor ofrezca oportunidades para aprender a autoevaluarse pues, como con otras habilidades, la práctica mejora esta capacidad. Desafortunadamente, es frecuente que los profesores se desanimen tras sus primeros intentos por conseguir este objetivo. Sin embargo, es importante tener en cuenta que la autoevaluación de una tarea como, por ejemplo, la realización de un resumen, es un aprendizaje complejo pues es preciso no sólo interorizar estrategias como la supresión de lo trivial, el uso de categorías supraordinales, de expresiones que engloben varias ideas, selección de la idea principal, sino también el modo de aplicarlas, y todo ello no se aprende de una vez. Además, es preciso tener presente 
que la autoevaluación no es una actividad que se produce en abstracto. Los criterios desde los que evaluar el propio trabajo varían en función de las tareas, por lo que no es fácil transferir lo aprendido cuando se cambia de tarea. En consecuencia, es necesario que los profesores no pierdan de vista que aprender a autoevaluarse y a autorregularse en el contexto de tareas concretas requiere primero, que el entrenamiento se realice en diferentes tareas -comprensión lectora, redacción, solución de problemas, etc.- y, segundo, tiempo.

5) Oportunidades para revisar y mejorar tanto el proceso de realización de la tarea como el resultado final. Una ayuda para estimular la autoevaluación es permitir a los alumnos que vuelvan a entregar sus trabajos si, al realizarla, detectan errores. En caso contrario, la autoevaluación pierde su valor, pues su objetivo es posibilitar la detección y corrección de los errores y la mejora del trabajo. Y si pierde su valor, la motivación de los alumnos por autoevaluarse se reduce, pues caer en la cuenta de los errores y no poder hacer nada por corregirlos produce impotencia (Pardo y Alonso-Tapia, 1990).

En resumen, son varias los aspectos pedagógicos que influyen en que la autoevaluación se produzca, y en cómo se lleva a cabo por parte de los alumnos. La variedad de posibilidades presentada puede llevar a pensar que es difícil enseñar a autoevaluar el propio trabajo, lo que no es cierto (Deakin-Crick, Sebba, Harlen, Guoxing, y Lawson, 2005). De hecho, algunas de las ayudas pedagógicas enumeradas -modelado, práctica, ofrecer pistas e instrucciones directas, etc.- son prácticas habituales en muchas aulas (Andrade y Valtcheva, 2009).

Uno de los aspectos claves para una buena implementación de la autoevaluación en el aula es la entrega de los criterios de evaluación, tal y como como se ha venido remarcando. Estos criterios se han ido entregando de diferentes formas por parte de los profesores a los alumnos a lo largo de los últimos años. Hay investigación que revisa estas formas más concretas que ha adoptado el entrenamiento de los alumnos en su autoevaluación (Brown y Harris, 2013), y se ha reunido abundante evidencia empírica usando la misma taxonomía que se presentará a continuación (Alonso-Tapia y Panadero, 2010; Panadero, 2011; Panadero et al., 2012; Panadero, Alonso-Tapia y Huertas, 2013; Panadero, Alonso-Tapia, Reche, 2013). A continuación pasamos a presentar estas diferentes opciones pedagógicas que se han usado para implementar la autoevaluación en el aula. 


\section{Ayudas instruccionales a la autoevaluación}

Atendiendo a revisiones bibliográficas, se han encontrado tres tipos de ayudas instruccionales a la autoevaluación dependiendo que los criterios de evaluación estén o no presentes y, en este caso, de cómo se presenten (Alonso-Tapia y Panadero, 2010; Panadero, 2011).

Entrenamiento en "autocalificación" o autoevaluación sin entrega de criterios de evaluación

Este procedimiento consiste en que al finalizar la actividad, el alumno evalúe y/o califique su trabajo sin que se le haya hecho entrega de los criterios de evaluación. Históricamente se ha considerado que autoevaluarse era lo mismo que autocalificarse -ponerse una nota-, concepción actualmente puesta en cuestión (Andrade y Valtcheva, 2009; Boud, 1995a). El mero hecho de pedir a los alumnos que se pongan una nota no garantiza que estos tengan en cuenta los criterios de evaluación y, por lo tanto, que valoren su trabajo de forma adecuada, lo que dificulta que el aulumo autorregule su trabajo de forma adecuada y que, en consecuencia, aprenda. De hecho, la evidencia empírica encontrada señala que la exactitud de las autocalificaciones es baja cuando los alumnos no conocen los criterios de evaluación por no haberse hecho explícitos (Tan, 2012).

\section{Entrenamiento mediante el uso de "rúbricas"}

Una rúbrica es un instrumento de autoevaluación y calificación que contiene los criterios de evaluación. Se compone de tres partes: Los criterios de evaluación, una escala para la autocalificación, y los indicadores de la calidad del trabajo (En la Figura 2 se muestra un ejemplo). La rúbrica resulta un instrumento útil porque explicita y ayuda a representarse el criterio de evaluación partiendo de modelos expertos, y a valorar el trabajo desde los indicadores de calidad que proporciona, si bien el grado en que ayuda depende de la precisión y calidad con la que esté confeccionada (Goodrich Andrade, 2001; Panadero, 2011). El uso de rúbricas está creciendo entre los países europeos y es una herramienta con una penetración alta y ya consolidada en EE.UU. 
Tabla 2. Rúbrica para evaluar el procedimiento de construcción de mapas conceptuales

\begin{tabular}{|c|c|c|c|c|}
\hline $\begin{array}{l}\text { Puntuación } \\
\text { Crit. de calidad }\end{array}$ & 4 & 3 & 2 & $\mathbf{1}$ \\
\hline Conceptos & $\begin{array}{l}\text { Contiene todos los con- } \\
\text { ceptos importantes y to- } \\
\text { dos los secundarios rele- } \\
\text { vantes }\end{array}$ & $\begin{array}{l}\text { Contiene los principales } \\
\text { y algunos secundarios, } \\
\text { pero faltan algunos se- } \\
\text { cundarios }\end{array}$ & $\begin{array}{l}\text { Contiene los conceptos } \\
\text { principales pero no los } \\
\text { secundarios }\end{array}$ & $\begin{array}{l}\text { Falta/n concepto/s prin- } \\
\text { cipales del tema }\end{array}$ \\
\hline Jerarquización & $\begin{array}{l}\text { Tiene una organización } \\
\text { correcta y completa, y el } \\
\text { mapa la transmite ade- } \\
\text { cuadamente }\end{array}$ & $\begin{array}{l}\text { La organización es co- } \\
\text { rrecta pero incompleta: } \\
\text { faltan niveles o elemen- } \\
\text { tos dentro de un nivel }\end{array}$ & $\begin{array}{l}\text { La organización es inco- } \\
\text { rrecta pero completa: hay } \\
\text { conceptos mal situados: } \\
\text { aparecen en un nivel dis- } \\
\text { tinto del que les corres- } \\
\text { ponde }\end{array}$ & $\begin{array}{l}\text { La organización es inco- } \\
\text { rrecta e incompleta: fal- } \\
\text { tan niveles o elementos } \\
\text { dentro de un nivel y otros } \\
\text { están mal situados }\end{array}$ \\
\hline $\begin{array}{l}\text { Relaciones entre con- } \\
\text { ceptos de diferente nivel } \\
\text { jerárquico }\end{array}$ & $\begin{array}{l}\text { RELACIONES } \\
\text { Son correctas: las líneas } \\
\text { unen los conceptos que } \\
\text { deben unir } \\
\text { NEXOS } \\
\text { Están explícitos y ayudan } \\
\text { a entender mejor las rela- } \\
\text { ciones }\end{array}$ & $\begin{array}{l}\text { RELACIONES } \\
\text { Son correctas pero in- } \\
\text { completas: faltan líneas } \\
\text { que deberían estar pre- } \\
\text { sentes. } \\
\text { NEXOS } \\
\text { Incompletos: Sólo se } \\
\text { explicitan algunos, pero } \\
\text { correctamente }\end{array}$ & $\begin{array}{l}\text { RELACIONES } \\
\text { Son parcialmente inco- } \\
\text { rrectas: algunas líneas } \\
\text { unen conceptos que no } \\
\text { deben sino } \\
\text { NEXOS } \\
\text { Incorrectos: Están todos, } \\
\text { pero algunos no son co- } \\
\text { rrectos }\end{array}$ & $\begin{array}{l}\text { RELACIONES } \\
\text { Son incorrectas en su } \\
\text { mayoría o inexistentes. } \\
\text { NEXOS } \\
\text { Incompletos e incorrec- } \\
\text { tos. }\end{array}$ \\
\hline $\begin{array}{c}\text { Relaciones entre con- } \\
\text { ceptos de distintas co- } \\
\text { lumnas } \\
\end{array}$ & $\begin{array}{l}\text { Están presentes y añaden } \\
\text { información importante }\end{array}$ & Sólo hay alguno & No hay & No hay \\
\hline $\begin{array}{c}\text { Simplicidad y facilidad } \\
\text { de uso }\end{array}$ & $\begin{array}{l}\text { Visualmente es sencillo y } \\
\text { claro. } \\
\text { Contiene ejemplos. }\end{array}$ & $\begin{array}{l}\text { Algunas líneas de rela- } \\
\text { ción no se entienden } \\
\text { Contiene sólo algún } \\
\text { ejemplo }\end{array}$ & $\begin{array}{l}\text { El número de conexiones } \\
\text { es excesivo y hace difícil } \\
\text { su uso } \\
\text { No contiene ejemplos. }\end{array}$ & $\begin{array}{l}\text { No se entienden bien las } \\
\text { relaciones ni la jerarquía } \\
\text { No contiene ejemplos. }\end{array}$ \\
\hline
\end{tabular}

Nota: aunque la nota máxima corresponda a 20 puntos se puede realizar un cálculo para calificar con un máximo de 10. 


\section{Entrenamiento mediante el uso de guiones de autoevaluación}

Los guiones de autoevaluación son conjuntos de preguntas organizadas sistemáticamente y centradas en el proceso de realización de la tarea (En la Figura 3 se muestra un ejemplo).

Tabla 3. Ejemplo guión de autoevaluación para la construcción de mapas conceptuales

\section{GUIÓN PARA AUTOEVALUAR \\ EL PROCESO DE CONSTRUCCIÓN DE MAPAS CONCEPTUALES}

1. ¿Me he hecho una pregunta de enfoque? (¿Tengo claro qué es lo que debe recoger el mapa conceptual?)

2. Basándome en esa pregunta, ¿he identificado conceptos suficientes para recoger las ideas del material trabajado?

3. ¿He ordenado los conceptos jerárquicamente poniendo los más amplios y globales al principio de la lista? (OJO: dependiendo de la naturaleza del mapa, al comienzo pueden ir conceptos concretos y específicos como, por ejemplo, si el mapa va a hablar del agua)

4. ¿He revisado la lista para añadir más conceptos si era necesario?

5. ¿He comenzado a construir el mapa con los conceptos más globales (o los conceptos clave a explicar) en la parte superior?

6. ¿He colocado 3 ó 4 sub-conceptos debajo de los más globales? Ten en cuenta que si tienes más de 6 conceptos en un mismo nivel es posible poner un concepto intermedio creando un nuevo nivel jerárquico (aunque no siempre es posible pues, por ejemplo, hay 8 clases de insectos).

7. ¿He unido los conceptos mediante líneas utilizando nexos -palabras que se ponen en las líneas para dar más información de la relación-?

8. ¿He dibujado líneas que relacionasen (vinculasen) conceptos situados en diferentes columnas de la jerarquía? Ayudan a descubrir nuevas relaciones.

9. ¿He puesto ejemplos bajo los conceptos para que fueran más sencillos de visualizar?

10. ¿He repasado el mapa conceptual una vez hecho teniendo en cuenta...

... que hay muchas formas de organizar los conceptos?

... cuál era el propósito perseguido y la información con que contaba?

La finalidad de los guiones es promover la reflexión sobre la adecuación del proceso que se va realizando y sobre los resultados parciales que se van obteniendo durante la realización de la tarea. Se ha comprobado que, en cuanto herramientas de autoevaluación, en algunos casos la eficacia de los guiones es incluso superior a la de las rúbricas (Panadero, AlonsoTapia y Huertas, 2012) Los guiones y los instrumentos afines (pistas e indicios -"prompts” y "cues" en inglés-) también permiten autoevaluar el trabajo al acabar, pero si solo se usan en ese momento, pierden parte de su potencial, pues los alumnos tendrían que hacer de nuevo la 
actividad en caso de detectar que algo es incorrecto al finalizar la tarea (Bannert, 2009; Thillmann, Kunsting, Wirth, y Leutner, 2009).

En resumen, tanto proporcionar rúbricas como guiones de evaluación son formas efectivas de fomentar la autoevaluación, pues proporcionan una base para saber cómo realizarla, lo que no ocurre cuando simplemente se dice a los alumnos que se autoevalúen. Ambos instrumentos, rúbricas y guiones, contienen los criterios de evaluación, aspecto que resulta fundamental para que tengan un impacto directo en la forma que los alumnos se autoevalúan. El uso de uno u otro depende de la intención pedagógica del profesor, que habrá de elegir cuál le resulta más adecuado para trabajar cada competencia (para más información, ver Panadero, 2011).

\section{Conclusiones}

El objetivo de este artículo era clarificar la complejidad del proceso de autoevaluación, y proporcionar indicaciones que facilitasen el trabajo de los profesores para promover su uso. Con el fin señalado se han hecho fundamentalmente tres cosas, cada una de las cuales constituye una aportación específica.

En primer lugar, se ha realizado una revisión de diferentes perspectivas teóricas sobre autoevaluación. Esta revisión ha puesto de manifiesto que el proceso de autoevaluación implica supervisar el propio trabajo desde los criterios de que se dispone para valorar su adecuación y, si procede, modificarlo. Forma parte integral, pues, del proceso de autorregulación que facilita el aprendizaje y la experiencia de progreso, experiencia que potencia la motivación por aprender. De hecho, una de las principales ideas que hemos querido transmitir es la importante relación existente entre la autoevaluación y la autorregulación. Si bien la autoevaluación por si misma es un proceso que ofrece mejoras en el aprendizaje de los alumnos si se hace una buena implementación de la misma (Brown y Harris, 2013), si se tiene en cuenta su importancia en la autorregulación y la activación de estrategias de aprendizaje, su importancia aumenta, máxime si tenemos en cuenta la abundante investigación que informa sobre los efectos educativos positivos de fomentar la autorregulación (e.g. Dignath et al., 2008; Paris y Paris, 2011). 
En segundo lugar, se ha puesto de manifiesto que, aunque la autoevaluación es importante para el aprendizaje y -según la investigación- es una práctica habitual en las aulas, sin embargo, muchos alumnos no autoevalúan su trabajo ni espontánea ni adecuadamente, bien porque no saben cómo - por ejemplo, por desconocimiento de los criterios específicos que deben tener en cuenta-, bien por que no ven qué valor puede tener realizar el esfuerzo que supone tal evaluación. Es necesario, pues, que los profesores enseñen de modo explícito a los alumnos a autoevaluar su trabajo. No obstante, un porcentaje alto de profesores no tienen clara cuál es su utilidad real -y por tanto, su importancia- ni cómo implementar prácticas que la favorezcan. Por esta razón en el presente trabajo, además de exponer en qué consiste y cuál es su relevancia para el aprendizaje y uso de estrategias por parte de los alumnos, se han expuesto e ilustrado algunos procedimientos que pueden utilizarse tanto para su enseñanza instrucción directa, modelado, etc.- como para motivar su utilización y facilitar su puesta en práctica -rúbricas y guiones fundamentalmente-. El profesor debe adaptar estos procedimientos al tipo de tarea -lectura, redacción, solución de problemas, realización de experimentos, etc.-, pues la autoevaluación y autorregulación no se producen en abstracto sino en el contexto de tareas específicas cuya adecuada realización responde a criterios diferentes.

Finlmente, se han revisado las condiciones que potencian la eficacia de la enseñanza de la autoevaluación. Primero, es recomendable permitir a los alumnos que puedan modificar sus trabajos si al autoevaluarse se dan cuenta de los errores, pues darles esta oportunidad les ayuda a ver su utilidad, experiencia que aumentará su motivación por autoevaluar su trabajo en el futuro. Y, segundo, hay que dejar que los alumnos practiquen para que puedan desarrollar su capacidad de autoevaluación en el contexto de tareas diferentes.

Resumiendo, la autoevaluación es una competencia crucial para el desarrollo de los alumnos, una competencia cuya adquisición se puede faciliar en el aula atendiendo a criterios pedagógicos y, lo que es también muy importante, sencillos de aplicar en nuestras aulas, como han demuestrado experiencias previas (e.g. Brown y Harris, 2013), 


\section{Referencias}

Alonso-Tapia, J. (2005). Motivar en la escuela, motivar en la familia. Madrid: Morata.

Alonso-Tapia, J., y Panadero, E. (2010). Effect of self-assessment scripts on self-regulation and learning. Infancia y Aprendizaje, 33(3), 385-397.

Andrade, H. (2010). Students as the definitive source of formative assessment: Academic self-assessment and the self-regulation of learning. In H. J. Andrade y G. J. Cizek (Eds.), Handbook of Formative assessment (pp. 90-105). New York: Routledge.

Andrade, H., y Valtcheva, A. (2009). Promoting learning and achievement through selfassessment. Theory Into Practice, 48(1), 12-19.

Bannert, M. (2009). Promoting self-regulated learning through prompts. Zeitschrift Fur Padagogische Psychologie, 23(2), 139-145. doi: 10.1024/1010-0652.23.2.139

Belfiore, P. J., y Hornyak, R. S. (1998). Operant theory and application of self-monitoring in adolescents. In D. H. Schunk y B. J. Zimmerman (Eds.), Self-regulated learning: From teaching to self-reflective practice (pp. 184-202). New York: Guilford Press.

Biemiller, A., y Meichenbaum, D. (1992). The nature and nurture of the self-directed learner. Educational Leadership, 50(2), 75-80.

Black, P., y Wiliam, D. (1998). Assessment and classroom learning. Assessment in Education: Principles, Policy and Practice, 5(1), 7-73.

Black, P., y Wiliam, D. (2009). Developing the theory of formative assessment. Educational Assessment, Evaluation and Accountability, 21(1), 5-31. doi: 10.1007/s11092-0089068-5

Boekaerts, M., y Cascallar, E. (2006). How far have we moved toward the integration of theory and practice in self-regulation? Educational Psychology Review, 18(3), 199210. doi: 10.1007/s10648-006-9013-4

Boekaerts, M., y Corno, L. (2005). Self-regulation in the classroom: A perspective on assessment and intervention. Applied Psychology-an International Reviewpsychologie Appliquee-revue Internationale, 54(2), 199-231.

Boud, D. (1995a). What is learner self-assessment? In D. Boud (Ed.), Enhancing learning through self-assessment (pp. 11-23). New York: RoutledgeFalmer.

Boud, D. (1995b). How does self-assessment relate to ideas about learning? In D. Boud (Ed.), Enhancing learning through self-assessment (pp. 24-35). New York: RoutledgeFalmer. 
Boud, D. (1995c). How can self-assessment be implemented? In D. Boud (Ed.), Enhancing learning through self-assessment (pp. 177-188). New York: RoutledgeFalmer.

Boud, D., y Falchikov, N. (1989). Quantitative studies of student self-assessment in highereducation: A critical analysis of findings. Higher Education, 18(5), 529-549. doi: 10.1007/BF00138746

Brown, G. T. L., y Harris, L. R. (2013). Student self-assessment. In J. McMillan (Ed.), The SAGE handbook of research on classroom assessment (pp. 367-393). Thousand Oaks, CA: SAGE.

Deakin-Crick, R., Sebba, J., Harlen, W., Guoxing, Y., y Lawson, H. (2005). Systematic review of research evidence of the impact on students of self- and peer-assessment Research Evidence in Education Library. London: EPPI-Centre, Social Science Research Unit, Institute of Education, University of London.

de la Fuente, J., y Justicia, F. (2007). The DEDEPRO Model for Regulating Teaching and Learning: recent advances. Electronic Journal of Research in Educational Psychology, $5(3), 535-564$.

de la Fuente, J., Zapata, L., Martínez-Vicente, J. M., Cardelle-Elawar, M., Sander, P., Justicia Justicia, F., Pichardo, M. C., y García-Berbén, A. B. (2012). Regulatory Teaching and Self-Regulated Learning in College Students: Confirmatory Validation Study of the IATLP Scales. Electronic Journal of Research in Educational Psychology, 10(2), 839866.

Deci, E. L., y Ryan, R. M. (1985). Intrinsic motivation and self-determination in human behavior. New York: Plenum.

Dignath, C., Büttner, G., y Langfeldt, H. (2008). How can primary school students learn selfregulated learning strategies most effectively? A meta-analysis on self-regulation training programmes. Educational Research Review, 3(2), 101-129. doi: 10.1016/j.edurev.2008.02.003

Dochy, F., Segers, M., y Sluijsmans, D. (1999). The use of self-, peer- and co-assessment in higher education. A review. Studies in Higher Education, 24(3), 331-350.

Goodrich, H. W. (1996). Student self-assessment: At the intersection of metacognition and authentic assessment. (57), ProQuest Information y Learning, US.

Goodrich, H. (2001). The effects of instructional rubrics on learning to write. Current Issues in Education 4(4). Retrieved from http://cie.ed.asu.edu/volume4/number4/ 
Greene, J. A., y Azevedo, R. (2007). A theoretical review of Winne and Hadwin's model of self-regulated learning: New perspectives and directions. Review of Educational Research, 77(3), 334-372. doi: 10.3102/003465430303953

Griffee, D. T. (1995). A longitudinal study of student feedback: Self-assessment, course evaluation and teacher evaluation. Alabama, US.

Kostons, D., van Gog, T., y Paas, F. (2009). How do I do? Investigating effects of expertise and performance-process records on self-assessment. Applied Cognitive Psychology, 23(9), 1256-1265. doi: 10.1002/acp.1528

Kramarski, B., y Michalsky, T. (2009). Three metacognitive approaches to training preservice teachers in different learning phases of technological pedagogical content knowledge. Educational Research and Evaluation: An International Journal on Theory and Practice, 15(5), 465 - 485.

Kramarski, B., y Michalsky, T. (2010). Preparing preservice teachers for self-regulated learning in the context of technological pedagogical content knowledge. Learning and Instruction, 20(5), 434-447. doi: 10.1016/j.learninstruc.2009.05.003

Kramarski, B., y Zeichner, O. (2001). Using technology to enhance mathematical reasoning: Effects of feedback and self-regulation learning. Educational Media International, $38(2), 77-82$.

Lan, W. Y. (1998). Teaching self-monitoring skills in statistics. In D. H. Schunk y B. J. Zimmerman (Eds.), Self-regulated learning: From teaching to self-reflective practice. New York: Guilford Press.

McCaslin, M., y Hickey, D. T. (2001). Educational psychology, social constructivism, and educational practice: A case of emergent identity. Educational Psychologist, 36(2), 133-140.

McCombs, B. L. (2001). Self-regulated learning and academic achievement: A phenomenological view. In B. J. Zimmerman y D. H. Schunk (Eds.), Self-regulated learning and academic achievement (pp. 67-124). New York: Lawrence Erlbaum.

Noonan, B., y Duncan, C. R. (2005). Peer and self-assessment in high schools. Practical Assessment, Research y Evaluation, 10(17), 1-8.

http://pareonline.net/getvn.asp?v=10yn=17

Panadero, E. (2011). Ayudas instruccionales a la autoevaluación y la autorregulación: Evaluación de eficacia de guiones de autoevaluación frente a la de las rúbricas. (Trabajo para la obtención del título de Doctor), Universidad Autónoma de Madrid, Madrid. 
Panadero, E. (En preparación). Survey on the implementation of formative assessment. A Spanish national study.

Panadero, E., Alonso-Tapia, J., y Huertas, J. A. (2012). Rubrics and self-assessment scripts effects on self-regulation, learning and self-efficacy in secondary education. Learning and Individual Differences, 22(6), 806-813. doi: 10.1016/j.lindif.2012.04.007

Panadero, E., Alonso-Tapia, J., y Huertas, J. A. (2013). Rubrics vs. self-assessment scripts: Effects on first year university students' self-regulation and performance. Infancia y Aprendizaje, 36 (3).

Panadero, E., Alonso-Tapia, J., y Reche, E. (2013). Rubrics vs. self-assessment scripts effect on self-regulation, performance and self-efficacy in pre-service teachers. Studies in Educational Evaluation. doi: 10.1016/j.stueduc.2013.04.001

Pardo, A., y Alonso-Tapia, J. (1990). Motivar en el aula. Madrid: Servicio de Publicaciones. Universidad Autónoma de Madrid.

Paris, S. G., y Paris, A. H. (2001). Classroom applications of research on self-regulated learning. Educational Psychologist, 36(2), 89-101.

Puustinen, M., y Pulkkinen, L. (2001). Models of self-regulated learning: A review. Scandinavian Journal of Educational Research, 45(3), 269-286. doi: $10.1080 / 00313830120074206$

Segers, M., Dochy, F., y Cascallar, E. (2003). Optimising new modes of assessment: In search of qualities and standards. Boston: Kluwer Academic Publishers.

Sitzmann, T., y Ely, K. (2010). Sometimes you need a reminder: The effects of prompting self-regulation on regulatory processes, learning, and attrition. Journal of Applied Psychology, 95(1), 132-144.

Tan, K. H. K. (2012). Student self-assessment. Assessment, learning and empowerment. Singapore: Research Publishing.

Thillmann, H., Kunsting, J., Wirth, J. y Leutner, D. (2009). "Is it merely a question of "What" to prompt or also "When" to prompt? The role of point of presentation time of prompts in self-regulated learning." Zeitschrift Fur Padagogische Psychologie 23(2): 105-115.

van den Boom, G., Paas, F., Merriënboer, J. J. G., y van Gog, T. (2004). Reflection prompts and tutor feedback in a web-based learning environment: Effects on students' selfregulated learning competence. Computers in Human Behavior, 20, 551-567.

Winne, P. H., y Hadwin, A. F. (1998). Studying as self-regulated engagement in learning. In D. Hacker, J. Dunlosky y A. Graesser (Eds.), Metacognition in educational theory and practice (pp. 277-304). Hillsdale, NJ: Erlbaum. 
Wirth, J. (2009). Promoting self-regulated learning through prompts. Zeitschrift Fur Padagogische Psychologie, 23(2), 91-94. doi: 10.1024/1010-0652.23.2.91

Zimmerman, B. J. (2000). Attaining self-regulation: A social cognitive perspective. In M. Boekaerts, P. R. Pintrich y M. Zeidner (Eds.), Handbook of self-regulation (pp. 1340).

Zimmerman, B. J. (2001). Theories of self-regulated learning and academic achievement: An overview and analysis. In B. J. Zimmerman y D. H. Schunk (Eds.), Self-regulated learning and academic achievement (Second ed., pp. 1-37). New York: Lawrence Erlbaum Associates.

Zimmerman, B. J., y Moylan, A. R. (2009). Self-regulation: Where metacognition and motivation intersect. In D. J. Hacker, J. Dunlosky y A. C. Graesser (Eds.), Handbook of Metacognition in Education (pp. 299-315). New York: Routledge. 